\title{
IdeAs
}

Idées d'Amériques

$12 \mid 2018$

Le tourisme dans les Amériques

\section{Tango et tourisme : dynamiques de valorisation et capitalisation à Buenos Aires}

Tango y turismo : dinámicas de valorización y capitalización en Buenos Aires

Tango and tourism: the dynamics of valorization and capitalization in Buenos

Aires

Linda Boukhris, Elsa Broclain, Sébastien Jacquot et Elodie Salin

\section{OpenEdition}

Journals

Édition électronique

URL : https://journals.openedition.org/ideas/3782

DOI : $10.4000 /$ ideas.3782

ISSN : 1950-5701

Éditeur

Institut des Amériques

Référence électronique

Linda Boukhris, Elsa Broclain, Sébastien Jacquot et Elodie Salin, «Tango et tourisme : dynamiques de valorisation et capitalisation à Buenos Aires », IdeAs [En ligne], 12 | 2018, mis en ligne le 06 novembre 2018, consulté le 20 octobre 2022. URL : http://journals.openedition.org/ideas/3782 ; DOI : https:// doi.org/10.4000/ideas.3782

Ce document a été généré automatiquement le 20 octobre 2022

\section{(c) $(1) \Theta($}

Creative Commons - Attribution - Pas d'Utilisation Commerciale - Pas de Modification 4.0 International - CC BY-NC-ND 4.0

https://creativecommons.org/licenses/by-nc-nd/4.0/ 


\title{
Tango et tourisme : dynamiques de valorisation et capitalisation à Buenos Aires
}

\author{
Tango y turismo : dinámicas de valorización y capitalización en Buenos Aires \\ Tango and tourism: the dynamics of valorization and capitalization in Buenos \\ Aires
}

Linda Boukhris, Elsa Broclain, Sébastien Jacquot et Elodie Salin

\section{Introduction}

1 Né dans le bassin du Rio de la Plata à la fin du XIX ${ }^{e}$ siècle, le tango fait l'objet d'une historiographie riche et controversée, souvent rédigée par des poètes (Ferrer, 1980 ; Salas, 1999) sous la forme d'une épopée des marges vers le centre, des bas-fonds vers l'élite (Patrix, 2014). La pratique du tango, relevant de la musique, de la danse et de la poésie, a été modelée par des métissages et des circulations, amenant Ramon Pelinski à la qualifier de "métaphore de la mondialisation" (Pelinski, 2011). Le tango a été diffusé à l'échelle internationale dès le début $\mathrm{du} \mathrm{Xx}^{\mathrm{e}}$ siècle, de l'arrivée des premiers musiciens et danseurs dans les sociétés mondaines européennes (Cooper, 1995 ; Zalco, 2016) au succès de Carlos Gardel dans les années 1930 (D'Iugo, 2009). À présent le tango se danse sur tous les continents, dans des milongas ${ }^{1}$ ou lors de festivals qui rassemblent parfois plusieurs milliers de personnes de Montréal à Tokyo, d'Istanbul à Tarbes. Mais cette dimension internationale reste structurée par la référence à ses lieux d'origine: Montevideo et Buenos Aires, considérée comme « la matrice du tango (Apprill, 2011). L'imaginaire transnational du tango intègre en même temps des marqueurs de la ville de Buenos Aires et les figures du tango sont abondamment reprises sur les guides touristiques consacrés à l'Argentine ou Buenos Aires. En 2009, suite à une présentation binationale entre l'Uruguay et l'Argentine, le tango est inscrit sur la liste du patrimoine culturel immatériel de l'UNESCO, dans une période marquée par le lancement de politiques publiques de valorisation, associées à une volonté de réancrer le tango dans 
le Rio de la Plata. À travers cette double politique patrimoniale et touristique, les Argentins cherchent à réaffirmer Buenos Aires comme capitale du tango, dans une logique d'ancrage des multiples circulations liées au tango (Debarbieux, Del Biaggio, Petite, 2008). Dans ce cadre, le tourisme est nourri par des stratégies de marketing territorial centrées sur le tango (Broclain, 2012), et soutenu par la promotion de TangoBA, un méga-événement international qui fusionne le Festival de tango et le Championnat Mondial.

2 Toutefois, il serait inexact de faire correspondre la naissance d'un tourisme du tango aux seules politiques publiques menées en ce début du troisième millénaire. Des initiatives privées existent depuis plusieurs décennies, notamment à partir du développement de spectacles-cabarets de tango à la fin des années 1960. Plus récemment, de nombreuses activités formelles et informelles se sont développées, sans lien nécessairement avec les politiques publiques, des écoles de danse aux visites guidées et accompagnement en milonga. L'association du tourisme et du tango se traduit par une pluralité d'initiatives, d'acteurs, de lieux, correspondant aussi à une pluralité des formes de tango.

3 Cet article interroge la relation entre tourisme et tango. Les liens entre les deux ont déjà été étudiés du point de vue des touristes et des circulations, et des économies de l'intimité auquel il donne lieu (Törnqvist, 2013; Apprill, 2012) en rapport avec des imaginaires globaux véhiculés par les mass medias (Fitch, 2015). Notre propos ici est décalé, considérant le tango au prisme des politiques publiques et des initiatives privées visant à sa valorisation, croisant les enjeux de la patrimonialisation sans postuler une prééminence patrimoniale (Gravari-Barbas, 2018), autrement dit questionnant la façon dont ces circulations interagissent avec des politiques localisées, entre enjeux marchands et de sauvegarde. Notre recherche explore alors ce décalage entre des interventions publiques destinées à accroître la valorisation touristique du tango, à partir de quelques actions ciblées de grande envergure (inscription unesco, politique événementielle) et la pluralisation des motifs et modalités de la pratique du tango, qui reconfigure aussi ce qui est identifié comme patrimoine local (Lacarrieu, 2006). Parallèlement, cet article examine la capitalisation du tango à Buenos Aires, au double sens d'une ville présentée comme capitale du tango, par des politiques explicites, et d'une activité génératrice de richesses et d'activité dans la ville.

4 Dans un premier temps, nous caractérisons la place du tango dans les fréquentations touristiques à Buenos Aires. Puis nous présenterons les acteurs hégémoniques d'un tango d'emblée touristique, entre cabarets-tango et politiques publiques soutenant des formes événementielles et d'envergure internationales. Toutefois, ces formes spectaculaires n'épuisent pas le tourisme tango, qui s'immisce aussi dans tout un ensemble de lieux diffus dans la ville, et génère de nombreuses activités de médiation, des cours aux accompagnements en milongas.

5 Cet article repose sur un recueil de données statistiques et bibliographiques ainsi que sur une centaine d'entretiens menés dans le cadre d'une recherche collective ${ }^{2}$, sur les liens entre patrimonialisation du tango et circulations. Les entretiens ont été menés avec les acteurs publics, intermédiaires touristiques, acteurs de la transmission (professeurs de tango, maestros), organisateurs de milongas, touristes et praticiens, à Buenos Aires mais aussi dans d'autres villes marquées par la présence d'une communauté tango (Paris, Nantes, Quebec, Nice, Valparaiso, Budapest), constituant un corpus de 80 entretiens. 


\section{Le tango, pratique touristique capitale à Buenos Aires}

6 L'Argentine a accueilli 7,2 millions de visiteurs internationaux en 2016 (WTO, 2017³), parmi lesquels un peu plus de 2 millions sont hébergés au moins une nuit dans un hôtel de Buenos Aires (Ente de Turismo, 2017). À ces touristes internationaux s'ajoutent les Argentins séjournant à Buenos Aires. Quelle place prend le tango dans leurs motivations et pratiques de visite sur place? Comment les imaginaires et prescriptions touristiques mettent en avant cette connexion entre Buenos Aires et le tango?

\section{Le tango dans les pratiques touristiques}

7 Le tango à Buenos Aires est pluriel dans ses géographies, ses activités et ses modes de structuration économique (carte 1). De nombreux quartiers sont concernés, du centre (San Telmo, San Nicolas, San Cristobal) aux quartiers péricentraux (La Boca, Abasto, Boedo) ou plus lointains (Villa Urquiza). La pratique touristique du tango consiste autant en un spectacle-cabaret qu'une virée nocturne en milongas, des cours particuliers, la visite du musée Gardel ou un city-tour à la Boca où se vendent cartes postales, tableaux et souvenirs thématisés tango. Aussi est-il difficile d'évaluer de façon simple sa place dans le développement touristique métropolitain, dans des statistiques locales du tourisme qui présentent surtout le tourisme par quartier ou secteur d'activité (hôtels, restaurants, etc) et qui questionnent rarement les pratiques touristiques.

\section{Carte 1 : Les géographies croisées du tourisme et du tango à Buenos Aires}

\section{Carte des auteurs}

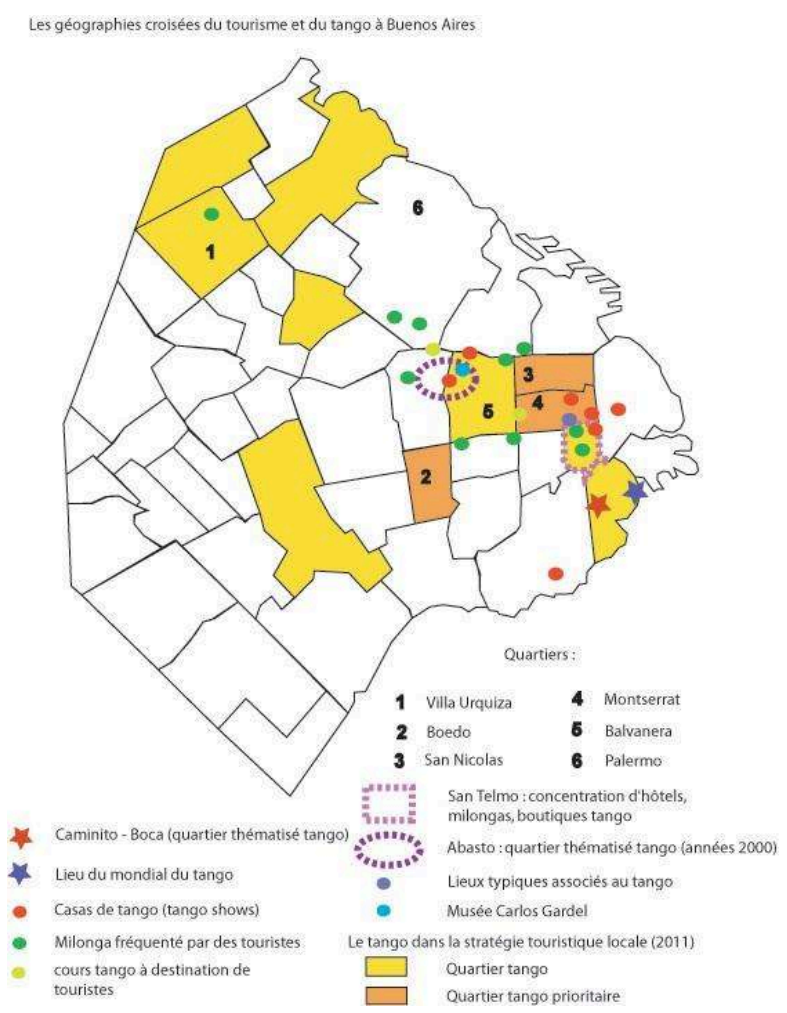


Deux enquêtes permettent de l'approcher statistiquement. Tout d'abord, les touristes internationaux sont interrogés aux points d'entrée du pays sur leurs motivations et pratiques (Ministerio de Turismo, 2016). En 2015, à partir de quatre portes d'entrées majeures (les deux aéroports de Buenos Aires, le port de Buenos Aires, l'aéroport de Córdoba), 21,8\% mentionnent des " activités liées au tango ", troisième réponse la plus fréquente après les activités culturelles en ville $(60,2 \%)$ et les expériences gastronomiques et œnologiques ( $44 \%$ ). Ces activités liées au tango concernent de façon préférentielle les touristes brésiliens (36,4\% des touristes interrogés en 2015), grands consommateurs des shows tango (cabaret tango), et les touristes d'Amérique du Nord (28\%). Cela est moins marqué pour les touristes provenant d'Europe (19\%), du Chili $(13,9 \%)$ ou du reste de l'Amérique latine (16,8 \%). Bien que très générale, cette enquête illustre ainsi des rapports différenciés au tango selon les provenances, et l'importance du tango dans le séjour argentin. Une autre enquête menée en 2006 sur la dimension économique du tango à Buenos Aires met l'accent sur l'importance des shows de tango dans l'expérience touristique, estimant qu'entre 15 et $40 \%$ des touristes internationaux à Buenos Aires assistent à un spectacle de tango durant leur séjour (OIC, 2007).

9 Des enquêtes menées à l'échelle locale et métropolitaine, notamment par l'Observatoire touristique de la ville de Buenos Aires (créé en 2009), permettent d'affiner ces analyses, bien qu'elles ne soient pas systématiques. Deux types de sources peuvent être utilisées : les enquêtes auprès des visiteurs lors du Mondial de Tango tous les ans en août, et d'autres enquêtes plus ponctuelles, par exemple sur la fréquentation du musée Carlos Gardel (situé dans la maison d'enfance du chanteur). Toutefois ces données concernent alors des pratiques de tango spécifiques, par des touristes pour lesquels la pratique du tango peut constituer un motif majeur du séjour.

10 Le Mondial de tango est supposé attirer environ 70000 touristes (jusqu'à 100000 certaines éditions selon les statistiques locales), restant en moyenne 3 jours dans la ville (anuario 2014). Pour l'édition $2014^{4}, 56 \%$ de ces touristes sont étrangers, provenant du monde entier ( $15 \%$ du Brésil, $37 \%$ du reste de l'Amérique centrale et du Sud, $21 \%$ d'Amérique du Nord, $15 \%$ d'Europe, $8 \%$ des autres continents). Une majorité d'entre eux vient avant tout pour le Mondial de tango. Evénement majeur financé par les acteurs publics de la culture et du tourisme, le Mondial révèle ainsi la dimension internationale de la pratique et le rôle de capitale du tango joué par la ville, polarisant des flux du monde entier.

11 Cet aspect est confirmé par les statistiques présentées par Tangotecnia, organisation argentine privée fondée par Jorge Arellano ${ }^{5}$, qui conduit depuis 2014 des enquêtes à partir de questionnaires diffusés via des groupes Facebook orientés tango dans le monde entier, concernant des praticiens (essentiellement des danseurs) et dont les résultats sont abondamment repris localement. L'échantillon d'enquête reflète ce mode de distribution, avec une répartition des enquêtés entre Argentine, Europe, Amérique du Nord et du Sud. Les résultats montrent la prééminence de Buenos Aires dans les géographies du tango. En effet, alors que les enquêtes portent sur le tango dans le monde, parmi les 41 milongas préférées les plus souvent citées, 31 sont localisées à Buenos Aires, les autres se trouvant au Brésil, Mexique, Allemagne, Angleterre, Uruguay ou ailleurs en Argentine. Une étude menée en 2017 est consacrée plus spécifiquement au tango comme motif de tourisme: si de nombreux voyages tango concernent l'Europe (festivals, etc.), l'Argentine apparaît comme la destination incontournable pour les danseurs, notamment dans le but de se perfectionner 
(TangoTecnia, 2017). Cette entrée par les études statistiques nous laisse ainsi entrevoir une pluralité de pratiques et nous éclairent sur les différentes modalités du rapport entre tourisme et tango à Buenos Aires.

\section{Les touristes à Buenos Aires, du dîner spectacle à l'immersion dans les mondes du tango}

Les façons d'expérimenter le tango à Buenos Aires définissent différents types de touristes. Ces types ne constituent pas des catégories figées, mais découlent des entretiens menés avec les acteurs et praticiens du tango, à Buenos Aires et dans des villes émettrices, en s'appuyant sur les qualifications et auto-qualifications qu'ils mobilisent.

Le touriste non initié au tango, qui vient à Buenos Aires pour la première fois et découvre la ville dans le cadre d'un circuit plus long en Argentine, ou lors d'un voyage d'affaires, constitue le public des tango shows. Les options pour découvrir la culture argentine à Buenos Aires sont, d'après les autorités en charge de la stratégie touristique, de deux ordres: la gastronomie et le tourisme expérientiel dans lequel le tango peut s'inscrire (Entretien avec un responsable d'une casa de tango, 2018). La soirée dans un tango show se déroule selon plusieurs modalités. Certaines casas de tango proposent un cours de tango avec les danseurs du spectacle avant le dîner, d'autres optent pour une dégustation de vins argentins, voire les deux comme à la Ventana à San Telmo où l'on peut choisir, en fonction de ses moyens et envies, entre trois spectacles différents (Entretien responsable clientèle La Ventana, 2018). Le dîner spectacle, tout compris, à l'image du Moulin Rouge à Paris se décline donc en plusieurs gammes de prix, des shows les plus exclusifs (Rojo Tango) aux plus économiques (au Tortoni). Certains spectacles optent pour une mise en scène spectaculaire façon Broadway comme Señor Tango fréquenté majoritairement par des Brésiliens, d'autres jouant la carte du classicisme (cabaret-tango Los Angelitos). Les hôtels et les agences de voyage sont démarchées par les casas de tango pour offrir leur produit en échange de commissions. Il s'agit d'ailleurs de la seule offre touristique spécifiquement tango proposée par les agences de voyage (Entretien Agence de voyages à Buenos Aires, 2018).

Figure 1 : Touriste et danseurs de tango sur la Plaza Dorrego

Elsa Broclain, 2018

IdeAs, 12 | 2018 


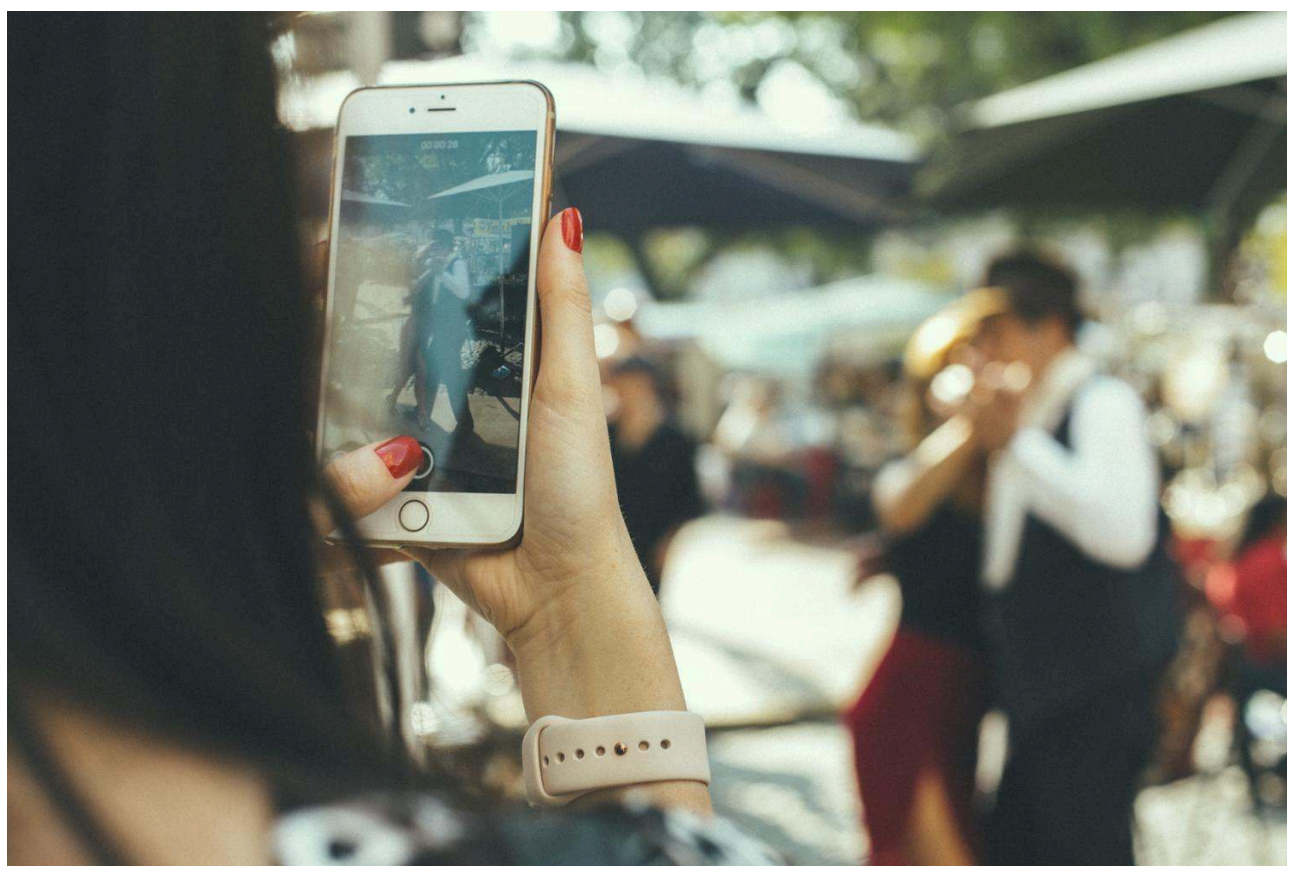

A l'opposé, ne fréquentant pas les cabarets tango, le touriste milonguero est un danseur plus ou moins expérimenté. Il reste plusieurs jours ou plusieurs semaines à Buenos Aires, voire plusieurs mois. Avec son sac de chaussures tango à la main, il sillonne Buenos Aires de milonga en milonga. Il compte apprendre, se perfectionner dans la danse en prenant des cours collectifs ou particuliers avec des maestros. Étranger, il aspire à danser et apprendre avec des Argentins, à vivre de façon immersive le tango. Il s'agit d'un touriste passionné, obsessionnel parfois, plusieurs de ces touristes assumant de ne pas visiter la ville durant leur séjour, et souvent ne se définissant pas comme " touriste " à l'image d'un couple de touristes suisses qui "reviennent dans le même hôtel boutique de Palermo depuis 17 ans pour danser le tango à la Viruta et au Salon Canning tout proches " (Entretien avec la propriétaire de l'hôtel boutique, 2018)Le témoignage d'un parisien danseur de tango souligne le côté addictif de la pratique du tango à Buenos Aires où il a déjà fait 5 séjours « $A$ chaque fois, je me disais que j'aimerais bien visiter l'Argentine, aller à Iguaçu, à Salta, et puis en fait, une fois arrivé à Buenos Aires, c'est quasiment impossible de s'en échapper (...) » (Entretien avec un danseur de tango, Paris, 2017). Ces voyages répétés peuvent se traduire par une installation à Buenos Aires, au motif du tango. L'imaginaire du tango de ces touristes milongueros est nourri de vidéos de danseurs connus (maestros), de références aux lieux et aux milongas qui forment comme un panthéon des lieux tango à pratiquer. Certains de ces touristes, timides ou inexpérimentés, passent parfois par des agences spécialisées ou par leurs professeurs européens qui organisent un voyage à Buenos Aires comme une étape incontournable de l'apprentissage du tango. "On a eu six clients de Tahiti une fois. Ces gens viennent vraiment pour le tango, trois semaines à fond, full day: cours particuliers, taxi-dancers, accompagnement en milonga, cours de technique féminine, de technique masculine... » (Entretien avec une danseuse de tango française ayant monté une agence de coaching tango pour les touristes, 2018).

D'autres parmi ces touristes milongueros sont des professionnels du tango en Europe ou en Amérique du Nord. Leur objectif est alors de prendre des cours avec les meilleurs maestros, de participer parfois au Mundial de tango afin d'acquérir reconnaissance et 
prestige. Le passage de ces professionnels (professeurs en Europe, musiciens, danseurs professionnels) entraîne parfois un séjour prolongé en Argentine.

Ce rapport à Buenos Aires comme capitale du tango n'est toutefois pas généralisé parmi les pratiquants tango dans le monde. Les «non-partants » (Duhamel, 2018), rencontrés lors de nos enquêtes à Paris, Nice, Nantes ou Québec, tout en ayant des mobilités liées au tango, refusent de passer par Buenos Aires qui ne serait plus d'après eux « la mecque du tango »: «Je n'ai pas eu envie d'aller à Buenos Aires. Des profs que je connais, ils reviennent (de Buenos Aires) avec une certaine suffisance (...) Tout le monde ne le fait pas en fait (le voyage à Buenos Aires), tout le monde n'est pas gangrené par cela » (Entretien avec une professeure de tango à Paris, 2016). Le refus d'un pèlerinage obligé à Buenos Aires est aussi justifié par la qualité et la diversité de l'offre du tango dans le monde. «Le meilleur niveau de tango, il est en Europe. Le problème en Europe, c'est qu'il y a beaucoup de technique et très peu d'émotion » (Entretien professeure de tango à Paris, 2016). Et si l'on dansait mieux le tango en Russie, en Corée, à Paris ou en Finlande qu'à Buenos Aires? La question, posée par plusieurs danseurs interviewés en Europe, à Buenos Aires, est corroborée par des articles de presse (Página 12, juin 2018). Le constat et les motifs invoqués sont la cherté de la vie à Buenos Aires pour les étrangers, le raccourcissement du séjour pour les milongueros, le fait qu'il existe des maestros (de Buenos Aires!) ailleurs dans le monde permettant un apprentissage de qualité et enfin la perte du monopole de Buenos Aires pour un tango exigeant dans les concours de danse. "Si tu veux de bons meneurs, tu vas en Turquie, les bonnes danseuses se trouvent en Russie, en Corée les danseurs sont extraordinaires et beaucoup de gens vont dans ces lieux, et encore d'autres plus incroyables comme en Méditerranée ou à Bali » (Página 12, Juin 2018). Buenos Aires, «la Reine du Rio de la Plata », n'a pas encore été détrônée, mais sa centralité n'est plus aussi évidente devant l'émergence de nouveaux pôles tangueros qui menacent son hégémonie.

Ces différentes pratiques de tango à Buenos Aires sont soutenues par des imaginaires multifocaux, relevant tant des guides touristiques ou de la promotion touristique que de la cristallisation d'imaginaires constitués par la circulation d'images et significations liées au tango via les mass media, depuis la première moitié $\mathrm{du} \mathrm{xx}^{\mathrm{e}}$ siècle, et qui articulent le tango à Buenos Aires ou explicitement à des formes de latinité.

\section{Les imaginaires du tango renvoient à Buenos Aires}

18 Les imaginaires du tango s'articulent autour de certains clichés et stéréotypes (Denigot, Mingalon, Honorin, 2015) tout autant circulant que les acteurs qui les portent (Fitch, 2015). Ils renvoient de façon nette à Buenos Aires, soutenant son rôle de berceau et capitale du tango. Le tango en devient emblème et marqueur urbain.

19 Ce sont d'abord les clichés liés à la narration des origines dans les bordels de Buenos Aires avec les figures de la prostituée et du mauvais garçon (portant chapeau et couteau) qui sont repris dans la plupart des tango show aujourd'hui. Cet imaginaire du tango s'enrichit aussi du glamour résultant du développement d'un tango mondain au début du $\mathrm{xx}^{\mathrm{e}}$ siècle, suite à la tangomania qui s'empare de l'Europe et plus particulièrement des lieux de la haute société ou des salons des stations balnéaires comme Deauville (Cooper, 1995). Le cinéma, notamment américain, participe activement à forger des images qui deviendront des lieux communs, telle la rose dans la bouche (Denigot, 2017), et propulse Carlos Gardel au rang de star internationale. La 
figure de ce dernier, qui symbolise le chanteur mythique de l'âge d'or du tango argentin, est abondamment reprise à Buenos Aires, aussi bien dans les spectacles tango que dans l'espace public (avec des fresques représentant Gardel à l'Abasto) ou avec la muséification de sa maison familiale. Combinant ces différents imaginaires, le récit enjolivé des origines, mis en scène dans les spectacles tango, positionne le tango dans le champ de la séduction, de la sensualité et lui ajoute une atmosphère nostalgique et une ambiance urbaine renvoyant aux bas-fonds de Buenos Aires, avec le lampadaire qui illumine une scène nocturne, les trottoirs ou les pavés des quais de la Boca.

L'étude des guides touristiques francophones et anglophones dédiés à l'Argentine et à Buenos Aires (46 guides de 2000 à $2010^{6}$ ) permet de retrouver ces clichés, associant une image globalisée du tango à Buenos Aires. Le tango constitue d'abord un des emblèmes de la destination argentine ou porteña. 24 couvertures sur 46 reprennent les imaginaires du tango (les 22 autres représentent des paysages naturels argentins ou la vie rurale symbolisée par le gaucho). L'image récurrente est celle d'un tango dansé avec le couple enlacé dans l'abrazo, suggestif (en ne montrant que les jambes), genré, coloré. Ces images sont évocatrices de la sensualité du tango, les danseurs se rapprochant dans un baiser suggéré. Des éléments du décor urbain viennent s'immiscer dans les couvertures et ces images centrées sur le couple s'élargissent alors de signifiants symbolisant l'espace urbain de Buenos Aires, notamment les façades colorées du quartier de la Boca ou les lampadaires de la ville.

Figure 2 : Couvertures des guides

De gauche à droite : Le Routard 2013, 2009, Le petit futé 2015, Le Routard 2016, National Geographic 2015, Lonely Planet 2008 et 2014, Cartoville, 2016.
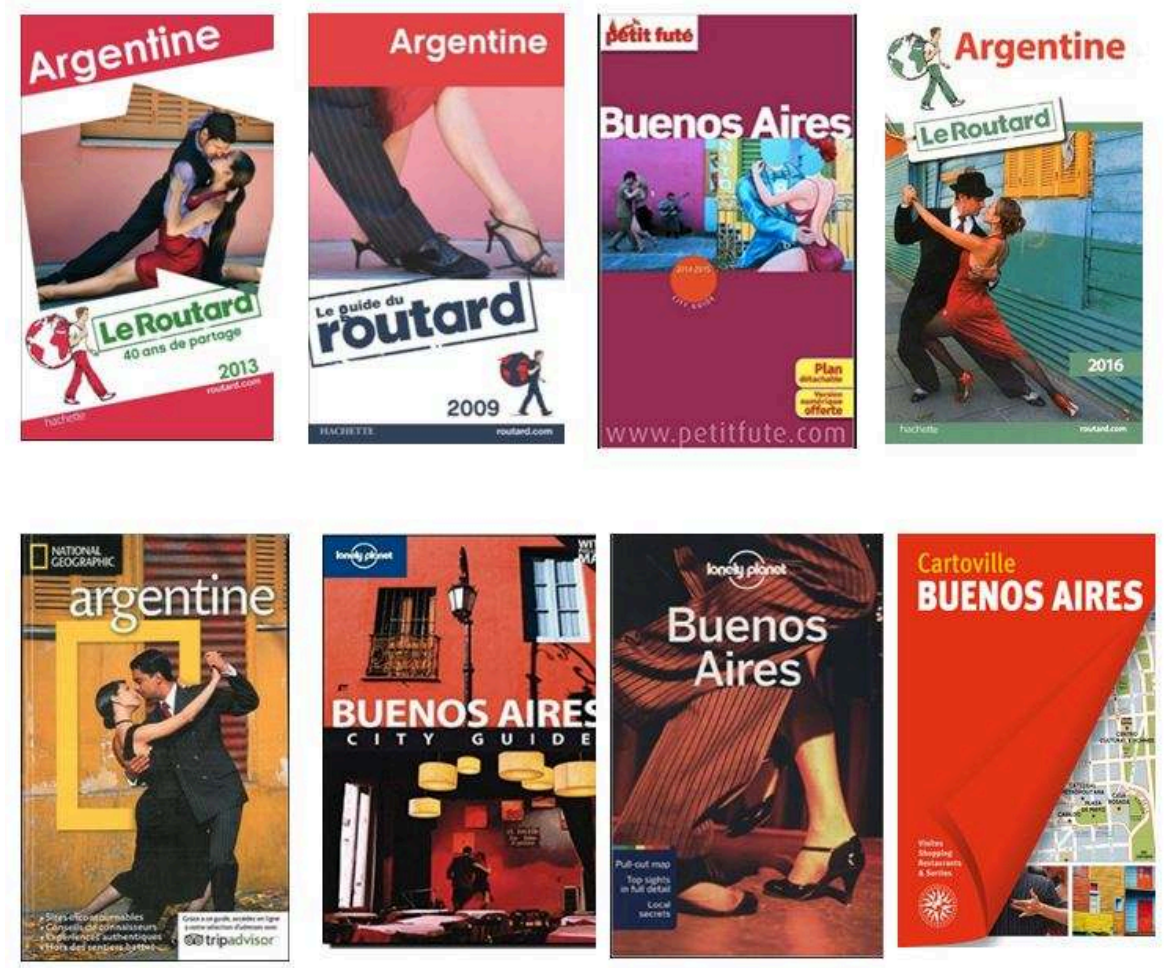

En outre, les guides contiennent des rubriques tango dans leurs pages sur Buenos Aires. «Les pieds s'entrelacent, les jambes s'enroulent, les corps s'emmêlent, les têtes 
chavirent ", peut-on lire dans le guide du Routard de 2016. Après un rappel de l'histoire du tango, les lieux emblématiques du tango sont proposés à la visite comme le Café Tortoni, la Confiteria Ideal (« Un des derniers grands cafés dansant de Buenos Aires »aujourd'hui fermé), les lieux "Où faire ses premiers pas, où pratiquer?", les bars tango, les milongas où «l'ambiance peut être moderne ou surannée, détendue ou traditionnelle » (Lonely Planet, 2017), sans oublier les lieux des spectacles de tango. Ces différents guides offrent ainsi un panorama de plus en plus exhaustif des lieux du tango à Buenos Aires, permettant à tous les touristes, des plus novices aux plus expérimentés dans le tango, de s'imprégner des imaginaires du tango et de parcourir les lieux dédiés (cafés, bars, milongas, casas de tango), les quartiers les plus représentatifs du tango (San Telmo, La Boca, Abasto), en proposant des dispositifs de visites (city-tours, circuits et visites accompagnés) pour danseurs expérimentés ou débutants avec des liens vers les agences réceptives spécialisées dans le tango.

Imaginaires globalisés et guides touristiques positionnent ainsi Buenos Aires en capitale du tango. Ce développement du tango apparaît toutefois structuré, tant par des politiques publiques de labellisation et promotion que des opérateurs privés développant les spectacles de tango, visant explicitement une internationalisation du tango en attirant des touristes étrangers, construisant un tango for export.

\section{Le tango comme produit touristique porteño}

Bien que la candidature UNESCO mentionne le Río de la Plata et tant Montevideo ${ }^{7}$ que Buenos Aires, les politiques de valorisation du tango concernent essentiellement la capitale argentine. Le tourisme tango à Buenos Aires est stimulé par les acteurs institutionnels, qui modèlent des politiques patrimoniales et touristiques, mais aussi par certains entrepreneurs, qui orchestrent le business du tango show. Ces deux types d'acteurs sont animés par des mouvements à la fois centripètes - ramenant l'ancrage (Debarbieux, 2014) du tango à Buenos aires - et centrifuges - exportant des shows dans le monde entier.

\section{Les politiques du tango et la réaffirmation d'un ancrage à Buenos Aires}

En 1996, la loi nationale du Tango (loi n 24.684) reconnaît « comme patrimoine culturel de la Nation la musique typique dénominée "tango", comprenant toutes ses manifestations artistiques telles que la musique, les paroles, la danse et les représentations plastiques qui y font référence». Son article 3 prévoit que les institutions chargées de la promotion de la culture et du tourisme à l'étranger incluent dans leurs programmes et brochures des références au tango "comme l'une des expressions culturelles typiques du pays ». Le tango y est présenté comme un référent identitaire national et la promotion touristique intervient pour le réancrer sur le territoire.

La création d'une nouvelle échelle de gouvernance territoriale replace toutefois le tango dans le giron local. Cette même année, Buenos Aires devient capitale fédérale, acquérant le statut de ville autonome, dotée d'un gouvernement et d'un budget propres. En décembre 1998, son assemblée législative vote la loi $n^{\circ} 130$ qui reconnaît cette fois le tango comme patrimoine culturel de la ville et institue la Fête populaire du 
tango qui deviendra le Festival TangoBA. Là encore, la loi inscrit la nécessité de "promouvoir la valeur touristique du tango », en collaborant avec le gouvernement fédéral.

À partir de la crise économique de 2001, le tourisme du tango est réinvesti comme un outil de résilience urbaine. Après la dévaluation de 2002, le boom touristique (Gorelik, 2006) généré par la conjoncture réoriente de manière décisive la politique de la ville qui mise alors sur ce flux de visiteurs pour accélérer une restructuration de l'économie postindustrielle. Le tango est alors en pleine expansion dans la ville et à l'étranger ${ }^{8}$. À Buenos Aires, le nombre de milongas serait passé de 27 en l'an 2000 à plus d'une cinquantaine en 2002 (La Nación, 17/03/2002), tandis que des festivals de tango fleurissent partout en Europe et que les boîtes de nuit internationales vibrent au son des tubes de tango électro du Gotan Project. En mars 2002, alors que le pays se relève à peine des événements de décembre liés à une aggravation de la crise économique et monétaire (el Argentinazo), le Secrétariat du Développement lance une enquête sur les touristes qui fréquentent les milongas, témoignant des vues des acteurs institutionnels sur la capacité du tango à générer des entrées de devises et relancer une économie en berne. Les politiques menées les années suivantes articulent les logiques de muséification (avec la création du musée-maison de Carlos Gardel en 2003) et de diffusion/valorisation (Championnat Mondial de tango, en 2003 également, cf. Morel, 2009), qui construisent simultanément le tango comme patrimoine et produit touristique.

Figure 3 : Le musée Carlos Gardel dans le quartier de l'Abasto à Buenos Aires

Elsa Broclain, 2018.

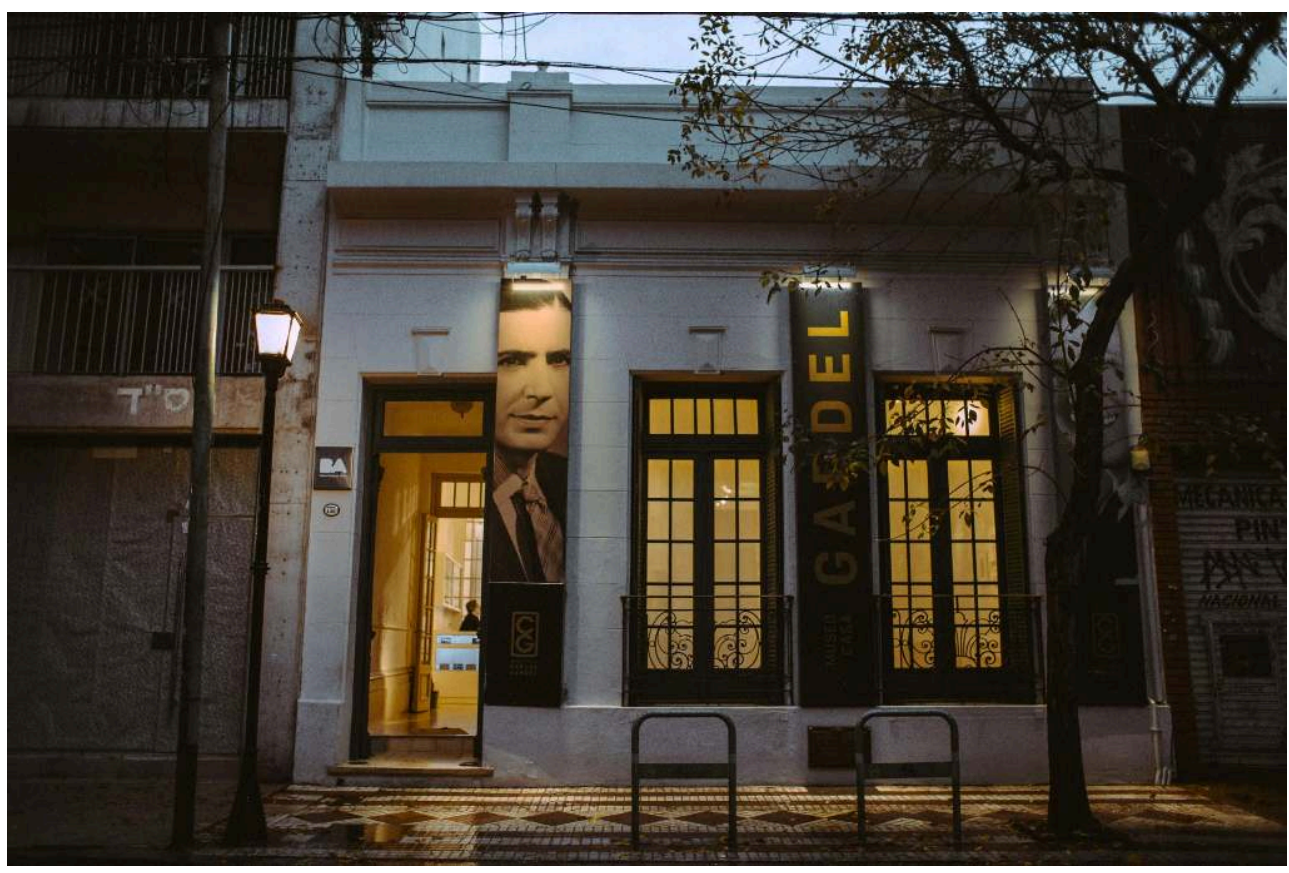

En 2007, le parti libéral-conservateur Propuesta Republicana (PRO) est élu à la tête du gouvernement local, et remet le tango au coeur des stratégies de développement territorial. Dans la perspective de couronner Buenos Aires capitale culturelle de l'Amérique Latine et en faire la promotion comme un pôle de tourisme culturel, le tango devient la figure de proue d'une politique de marketing urbain. Quand la crise de 
2008 amorce une contraction du tourisme dans le monde, le tango promu «marque ville » doit alors devenir " el gran invitador », c'est-à-dire un motif majeur d'attraction de visiteurs étrangers et "la plateforme de lancement de la ville à travers le monde", d'après le ministre de la Culture Hernán Lombardi (El Mundo,12/08/2009). Cet ancien promoteur hôtelier a organisé une synergie entre le ministère de la Culture et el Ente de Turismo, un nouvel organisme public-privé destiné à piloter le tourisme. Sa stratégie s'articule sur trois axes principaux : affirmer l'identification entre la ville et le tango, organiser un calendrier culturel en fonction des besoins de relance touristique, et promouvoir la ville à l'étranger à travers la production d'événements tango. Sa première mesure consiste à instaurer un "mois du tango ", en déplaçant le festival international sur les deux dernières semaines d'août et en le fusionnant avec le championnat Mondial de tango, afin de créer un méga-événement capable de stimuler les flux de visiteurs étrangers en basse saison.

La ville de Buenos Aires, dans son plan stratégique 2011-2015, consolide cette place du tango dans le développement touristique, en en faisant un des " produits » majeurs du développement et de la diffusion territoriale du tourisme. Parmi les 48 quartiers de la ville, 23 sont l'objet d'une stratégie détaillée et le tango y est mentionné pour 11 de ces quartiers, dont 4 fois de façon prioritaire. Plus récemment, la ville de Buenos Aires a refondé toute sa communication touristique, qui passe désormais par une application pour smartphone, et le tango (avec le fileteado) constitue l'une des 6 entrées thématiques de l'ensemble de l'offre touristique.

Parallèlement, le ministère présente un dossier pour inscrire le tango sur la liste du Patrimoine culturel immatériel, dernière étape d'un processus initié en 1990 par la création de l'Académie nationale du tango. Devant la mise en concurrence de Buenos Aires avec de nouveaux pôles tangueros dans le monde, la candidature du tango à l'Unesco permettait de revendiquer la centralité et l'authenticité d'un tango du Rio de la Plata contre "l'image déformée » qui en est montrée à l'extérieur (Unesco, 2009). Cette initiative reflète une stratégie de reterritorialisation du tango à Buenos Aires (Gómez Schettini, Almiron, González Bracco, 2011), pour lutter contre d'autres logiques de globalisation de la pratique.

Dans un mouvement inverse et complémentaire, le ministère finance des shows de tango exportés à l'étranger à des fins de promotion territoriale. En octobre 2013, la coproduction du spectacle Chantecler en résidence pendant près d'un mois au Théâtre du Châtelet s'inscrivait dans cette démarche. En plus des représentations, la troupe dirigée par la danseuse Mora Godoy proposait des cours d'initiation au tango, tandis que le personnel du ministère de la Culture porteño remettait des brochures sur Buenos Aires au public et un questionnaire interrogeant ses perceptions de la ville. Telle qu'elle est pensée par le gouvernement du PRO, la promotion du tango ne constitue pas un but en soi mais un moyen de renforcer la compétitivité de la ville face à d'autres destinations du tourisme culturel, en s'appuyant sur les spectacles de tango à Buenos Aires et exportés à l'étranger.

\section{Les tango show, de Buenos Aires vers le monde.}

31 Ces politiques publiques rebondissent sur l'important développement de l'offre de shows de tango à partir des années 2000. 

tango ne sont pas une invention récente, les liens entre tango et spectacle remontant même à la fin du xIX siècle. À cette époque, les musiciens argentins circulent entre les mondes du cirque et du théâtre, et jouent parfois des tangos dans les saynètes, de courtes pièces de théâtre musical qui évoquent la vie des habitants des conventillos. Le tango social s'invente en même temps qu'il est mis en spectacle, devenant l'attraction des cabarets et cafés-concerts qui bourgeonnent à Buenos Aires dès le début du siècle. Certains artistes vont tenter leur chance en Europe et aux Etats-Unis, et participent à la diffusion de la danse et de la musique, qu'ils adaptent au goût du public local. Les performers mélangent les figures de tango avec les danses de variété en vogue : cakewalk, danse apache, n'hésitant pas à se vêtir de gauchos et intégrer des numéros de boleadores qui s'inspirent des films de Rudolph Valentino. En 1920, Medrano, fameux danseur de tango à New York, raconte à la revue Caras y Caretas qu'il aurait préféré danser avec un masque au cas où un compatriote argentin l'aurait surpris en train de commettre son "attentat tanguiste " (Matallana, 2013). Le tango for export s'élabore dans cet aller-retour culturel entre les cabarets de Pigalle et de Broadway, mettant en scène une identité argentine exotisée nourrie par les représentations cinématographiques. Argentins qui se tournent vers d'autres genres plus à la mode comme le folklore ou le rock (Pujol, 1999). En pleine décroissance, le monde du tango voit alors dans le tourisme une opportunité pour se réinventer. Les étrangers mélomanes viennent écouter les grands du tango qui se produisent encore dans quelques salles prévues pour un public aisé, tels le Club 676 ou Caño 14, qui accueillent aussi les démonstrations de danse de Juan Carlos Copes et Maria Nieves. Les premiers spectacles tango au tournant des années 1960 et 1970 sont d'abord pensés à la fois pour les locaux et les touristes, puis finissent par se diriger explicitement vers le public international à l'image du Bar Sur (1967) ou du Viejo Almacen (1969). Les danseurs et musiciens rallient des compagnies et survivent grâce à des tournées internationales articulées sur trois régions: les Etats-Unis, l'Europe et le Japon. Parmi ces tournées, celle de Tango Argentino étrenné au théâtre du Châtelet, à Paris, en 1983 puis à Broadway en 1985, est considérée comme le point de départ d'une renaissance du tango à l'étranger.

Figure 4 : Tango Porteño, l'une des principales casas de tango 


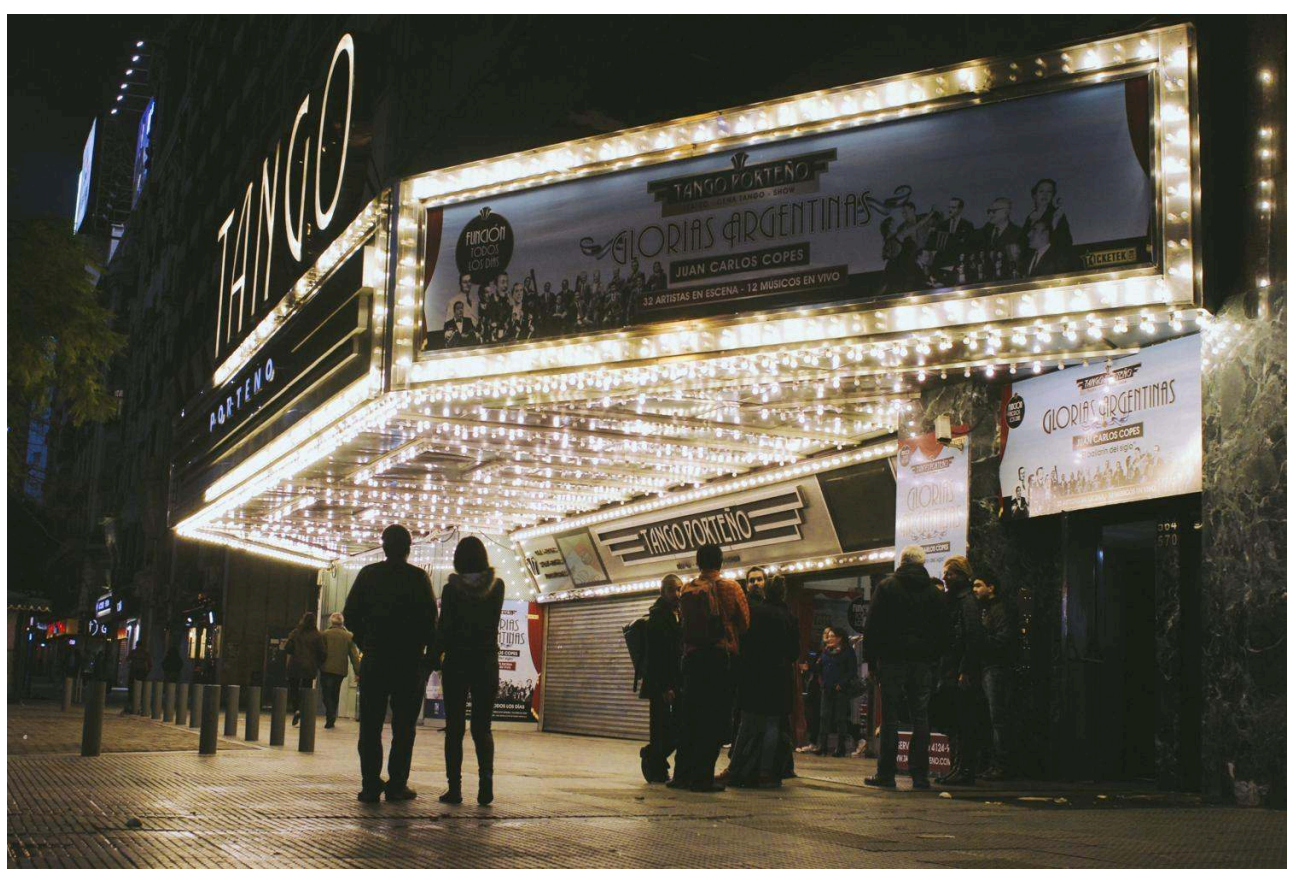

Entre 2003 et 2006, l'offre de "spectacles internationaux » aurait augmenté de $35 \%$ par an, d'après une étude de l'Observatoire des industries culturelles (OIC, 2007). Selon cette même étude, les dîners-shows de tango constituent l'une des activités touristiques principales des visiteurs étrangers à Buenos Aires, et surtout la plus rentable, représentant près de la moitié des gains perçus par l'industrie du tango (OIC, 2007). Ce secteur du tango spectacle se partage entre les tanguerias locales et les shows exportés dans le monde entier, notamment en Russie, au Japon, en Chine et au Brésil, où chaque année des compagnies tournent pendant plusieurs mois consécutifs. À l'exception de quelques spectacles qui construisent une narration proche de la comédie musicale, la plupart des shows se compose d'une succession de tableaux qui présentent de façon chronologique différentes époques du tango. D'après Juan Fabbri, propriétaire de trois casas de tango et d'une compagnie internationale :

En vérité, peu importe ce qu'est le tango. Ce qui importe c'est ce que les gens imaginent qu'est le tango, et c'est cela que tu dois livrer, parce qu'on parle d'un show pour touristes. C'est autre chose si je vais danser au Sin Rumbo'. Il faut différencier le tango social du tango spectacle. (Entretien, 2015)

Selon lui, la clé de son succès réside dans l'anticipation des attentes du public lorsqu'il exporte son show: exacerbant la sensualité et l'érotisme pour le public brésilien, la virtuosité et la technique pour les Russes, une élégance passionnée mais pudique pour les théâtres de Dubaï. Invitées par l'Institut national de promotion touristique (INPROTUR), les casas de tango participent systématiquement aux salons internationaux de tourisme, travaillant main dans la main avec les différents bureaux de l'administration du tourisme. Les politiques de promotion touristique du tango prennent ainsi le parti de mettre en valeur les shows for export, tandis que les autres secteurs bénéficient des retombées du festival TangoBA et de l'effet de vitrine produit par la mise en marque du tango. 


\section{Scènes et arrière-scènes du tango : un écosystème polycentrique}

L'écosystème patrimonial et touristique du tango ne peut se résumer à ces acteurs institutionnels qui façonnent l'image internationale de Buenos Aires comme capitale du tango, ni même à ces acteurs privés qui capitalisent sur une certaine image du tango dans le cadre notamment des tango shows. Il existe en effet une diversité d'acteurs impliqués dans la valorisation patrimoniale et touristique du tango à Buenos Aires, témoignant de la complexité d'un écosystème davantage polycentrique. Cette multiplicité d'entrepreneurs du tango à Buenos Aires reflète une pluralité de représentations, de pratiques et de lieux du tango. L'analyse de ces acteurs révèle par ailleurs une porosité du champ patrimonial et du champ touristique dans lesquels ces derniers s'inscrivent, déploient leurs logiques entrepreneuriales, légitiment leurs positionnements et/ou portent leurs revendications.

\section{Les entrepreneurs touristiques du tango : logiques micro- entrepreneuriales et globalisées}

Les entrepreneurs du tango s'inscrivent dans l'ensemble des secteurs de l'économie du tourisme offrant une thématisation et des produits touristiques articulés autour du tango. Il existe ainsi des hébergements touristiques proposant une thématisation des chambres autour de l'imaginaire du tango, des enseignements personnalisés ou collectifs de tango dans un espace dédié de l'hôtel ou encore des accompagnements nocturnes dans les milongas de Buenos Aires.

L'hébergement touristique offre ainsi une continuité de l'expérience tango des visiteurs et participe activement de la valorisation touristique du tango. La Antigua Casa de Brigit à San Telmo est un exemple d'hébergement touristique de type maison d'hôtes (ouverture des chambres sur un patio central) relié en partie au tango. Des cours privés à la demande sont donnés dans le patio. L'hébergement figure sur le catalogue en ligne d'une agence allemande spécialisée dans les voyages tango. Les habitués viennent du monde entier (Australie, Nouvelle-Zélande, Allemagne, Suisse...) dans cette pension coquette tenue par une Suissesse ayant refait sa vie à Buenos Aires grâce au tango. Ces touristes du tango sont des repeaters pour la plupart et restent plusieurs semaines sur place, en couple ou entre amis, parfois seuls. L'idée de la propriétaire est d'aider les femmes de plus de 50 ans dans leur découverte du tango à Buenos Aires. Forte de son expérience, elle tente de les orienter par ses conseils ou en leur recommandant des taxidancers de confiance (Entretien avec la propriétaire de la pension, 2017). Plus ancien, l'hôtel Malabia à Palermo accueille aussi une clientèle fidèle de tangueros internationaux sans pour autant l'exposer sur son site web. Plutôt chic, cet hôtel boutique situé dans un ancien couvent du $\mathrm{XIX}^{\mathrm{e}}$ siècle réhabilité est un des premiers « hôtel culturel » situé à Palermo Soho alors que le quartier n'était pas encore gentrifié. Il se situe à proximité de célèbres milongas : Salon Canning, La Viruta et la Catedral. Environ 100 personnes par an reviennent à l'hôtel boutique pour le tango.

Cette économie du tango s'articule également autour de nombreuses initiatives entrepreneuriales ciblées sur l'enseignement du tango que ce soit dans le cadre d'établissements spécialisés à l'image de DNI, qui organise des cours collectifs et 
particuliers pour tout type de public dans un lieu convivial (cafétéria, boutique d'article de tango DNI, salle de cours tous niveaux, organisation d'un bal d'entraînement avant la milonga (practica), ou dans le cadre de cours particuliers portés par des professeurs de tango. "A DNI, on fait un travail profond et dans la durée, de 1 à 5 mois pour certains. C'est sympa, on crée une communauté de connaissances. " (Entretien avec un touriste français élève à DNI et en année sabbatique en Argentine, 2017). Les professionnels du tango publicisent leur offre via des flyers distribués dans les lieux touristiques, les hôtels, les milongas et font l'objet d'une communication sur Tripadvisor, les réseaux sociaux, les sites Internet créés spécifiquement. Dans le cas de DNI, les réseaux sociaux, le nombre de participants dont beaucoup d'étrangers, la renommée de sa propriétaire, Dana Frigoli, professeure, danseuse, chorégraphe et entrepreneure, la présence d'une compagnie de danse composée par les professeurs de l'école et des prix attractifs contribuent au succès du modèle.

Plus récemment, certains enseignants relaient leur offre via la plateforme Airbnb Experience. La pluralité de ces produits à destination des touristes pousse également les enseignants à développer leur offre d'enseignement en direction d'une expérience tango qui se veut immersive et totale. Cette expérience tango peut prendre la forme d'une balade urbaine à travers le regard du professeur, à la découverte des lieux tango, du café notable à la milonga de quartier. Il ne s'agit plus alors d'apprendre le tango mais de vivre l'expérience tango à Buenos Aires, sous la forme d'un produit touristique complexe. C'est dans cette démarche que s'inscrit l'agence Tango Taxi Dancer, créée en 2007 par un professeur de danse argentin et reprise par une praticienne de tango germano-italienne installée à Buenos Aires depuis 2012. Celle-ci a commencé à danser le tango à Munich en 2009 en travaillant pour une professeure de tango argentine. Après avoir fondé l'agence "Tango project» chargée d'organiser les cours des professeurs de tango argentins à Munich, organisé un festival de tango et géré trois milongas à Munich, elle s'installe à Buenos Aires avec son ex-mari fondateur de l'agence de taxi dancers qui propose des services de danse aux touristes pour apprendre le tango et le pratiquer avec des danseurs professionnels en milonga. La différenciation de l'offre d'enseignement s'opère ici dans la pratique du tango en milonga avec des danseurs professionnels puisqu'il s'agit de se rendre in situ en milonga et danser le tango comme un milonguero. La dimension expérientielle est d'ailleurs relevée dans les commentaires des clients, par exemple sur Tripadvisor : «an excellent guide for a fabulous Buenos Aires Tango experience». Son agence est composée de 4 hommes dont 2 professeurs et 4 femmes dont 2 professeures. L'entrepreneure a déposé la marque Tango Taxi Dancer.

41 À l'instar de la démarche de Tango Taxi Dancer, une grande partie des acteurs touristiques qui composent l'écosystème du tango à Buenos Aires hors tango shows s'inscrivent dans une logique micro-entrepreneuriale, c'est-à-dire basée sur des initiatives individuelles ou en partenariat et disposant de peu de capitaux au départ. Dans le cadre de Tango taxi Dancer, c'est le salon de l'appartement de la directrice de l'agence située à San Telmo qui fait office de salle de cours. Mais certains de ces acteurs correspondent à des profils d'entrepreneurs multi-situés et globalisés qui s'adressent à une clientèle internationale, circulant à Buenos Aires, recyclant en compétences leurs expériences de circulations, antérieures à leurs activités ou encore situées au cœur de celles-ci. En effet, nombre de ces entrepreneurs touristiques sont soit des étrangers (Européens, Nord-américains...), soit des Argentins ayant voyagé, mobilisant leur «argentinité » et renforçant un capital social composé d'un réseau de contacts à 
l'international ainsi qu'un capital symbolique caractérisé par la constitution d'une réputation au sein de la scène tango.

Si ces circulations semblent constituer un élément central dans l'écosystème du tango, elles ne sont toutefois pas systématiques et nécessaires, à l'image de nombreux acteurs locaux de la valorisation patrimoniale du tango.

\section{Le réseau des milongas : hybridation patrimoniale et touristique}

Ces logiques entrepreneuriales s'appuient également sur un réseau de milongas dont la géographie témoigne d'une territorialisation diffuse du tango à Buenos Aires, dans différents quartiers. Chaque soir, les milongas deviennent des centres névralgiques de la pratique et culture tango de la ville. Celles-ci présentent une variété de profils dans les représentations et les pratiques du tango véhiculées, des plus conventionnelles aux plus alternatives. S'il existe plus d'une cinquantaine de milongas à Buenos Aires, quelques-unes d'entre elles sont devenues de véritables institutions au sein de la géographie urbaine du tango: La Viruta, Salon Canning, La Catedral, Club Gricel, La Marshall, etc. Chacune d'entre elles présente ses propres caractéristiques en matière de musique (musiciens ou DJ, genre musical), de style de danse, de type de publics (locaux, étrangers, jeunes, seniors etc.) et de philosophie générale à l'égard du tango. Certaines font l'objet d'une véritable spécialisation à l'instar des milongas La Marshall ou la Milonga Tango Queer avec un public de praticiens de tango majoritairement issus de la communauté LGBTQ.

Ce réseau de milongas est pénétré de façon croissante par les logiques touristiques et est intégré par les entrepreneurs du tango à l'élaboration du produit touristique à l'image de l'agence Tango Taxi Dancer. Certains hôteliers interrogés expliquent également la localisation de leur hébergement touristique à proximité d'une milonga comme un facteur déterminant de leur investissement, garantissant l'attractivité nécessaire à leur activité. Les organisateurs de milongas sont également des acteurs clés dans la valorisation touristique du tango. De nombreuses milongas proposent des cours en plusieurs langues, recrutent des "taxi dancers" afin d'attirer un public étranger ou encore collaborent avec des agences et écoles de danse pour recevoir des touristes danseurs en apprentissage. La milonga Viva la Pepa du Club Malcolm illustre à cet égard un profil d'organisateur de milonga intégrant de façon stratégique la logique touristique. L'organisatrice de cette milonga, Pepa Palazon, souhaite attirer des touristes et des locaux, sans chercher à opposer ces deux types de publics (Entretien avec Pepa Palazon, 2018). Elle présente en effet sa milonga comme «underground » et touristique et gère le lieu comme un véritable business tout en étant portée par sa passion pour le tango qu'elle décrit de façon lyrique. Elle anime plusieurs pages Facebook dont elle gère finement la fréquentation de ses dizaines de milliers d'abonnés et une chaine youtube. Elle développe une stratégie évènementielle afin d'accroître la fréquence et la popularité de la milonga dans le cadre d'une stratégie de différenciation, à l'image du "Mundialito", concours proposé quinze jours avant le Mondial du tango, qui se veut alternatif et populaire.

Enfin, plusieurs touristes interrogés déclarent consulter le site Hoy Milonga qui énumère quotidiennement les milongas de Buenos Aires. Le site est en anglais et témoigne ainsi de la pénétration de la logique touristique dans ces espaces dédiés à la pratique du tango. 


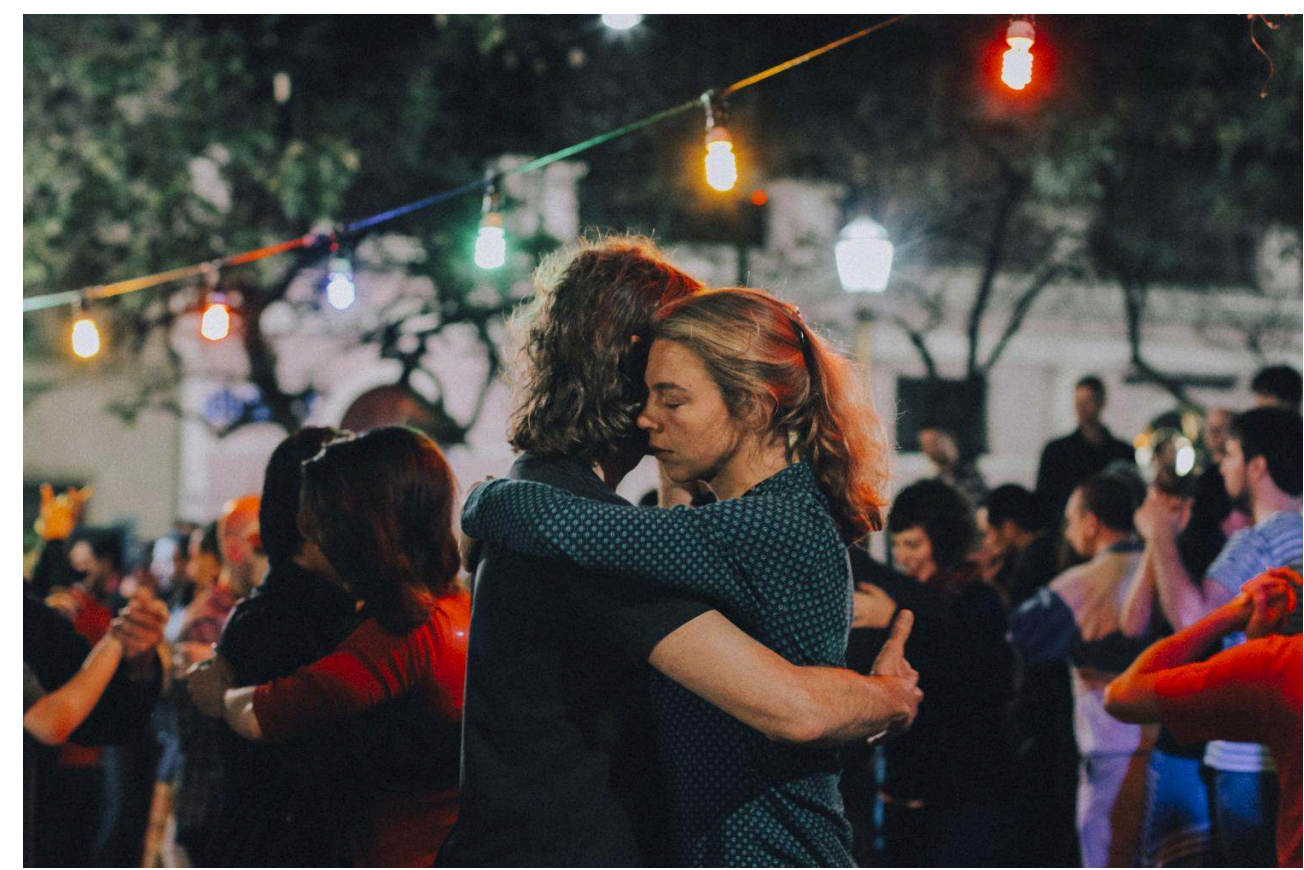

Le réseau des milongas, ses acteurs et les espaces d'interactions qu'elles offrent sont ainsi au cœur de cet écosystème polycentrique. Toutefois il ne faudrait pas en déduire une pluralité d'opportunités convergentes. De nombreuses milongas font régulièrement l'objet de menaces de fermeture pour raisons administratives ou sont très largement freinées dans leurs activités par un manque de soutien matériel et financier, alors même que le patrimoine culturel porteño fait l'objet d'une large communication institutionnelle. C'est dans ce contexte qu'un mouvement est né à Buenos Aires visant à contester ces fermetures jugées abusives par les organisateurs de milongas et en contradiction avec la politique de promotion du tango de la ville, à l'image du groupe «El tango no se clausura » créé par des milonguera/os de Buenos Aires. La milonga del Indio, qui se déroule chaque dimanche soir sur la Plaza Dorrego dans le quartier de San Telmo est un exemple de cette défense d'un tango dit social. L'organisateur de cette milonga fait partie de l'association des organisateurs de milongas de Buenos Aires et développe un discours critique à l'égard du manque de soutien institutionnel dans la valorisation patrimoniale du tango. Il fait de sa milonga à ciel ouvert un espace de lutte et de revendication, tandis que la forte présence touristique à sa milonga au cœur du quartier historique de la ville constitue à la fois l'occasion de promouvoir de façon large sa vision du tango comme patrimoine culturel et une forme de légitimation de sa présence.

47 Ces arrière-scènes du tango que constituent les milongas sont ainsi plurielles et s'inscrivent dans des dynamiques complexes qui témoignent de l'imbrication des logiques de valorisation patrimoniale et touristique du tango. 


\section{Conclusion}

La double politique patrimoniale et touristique des autorités de Buenos Aires a permis, depuis une décennie, de réinscrire le tango dans l'agenda des touristes de Buenos Aires. Qu'ils soient aficionados ou amateurs, simples spectateurs de tango shows ou professionnels du monde entier venant pour le Mundial de Tango, leurs mobilités et pratiques liées au tango s'imprègnent et percolent avec les imaginaires d'un Buenos Aires fantasmé et idéalisé. Néanmoins, le tango pratiqué aujourd'hui dans le monde entier bénéficie d'une image transnationale bien réelle au delà des frontières argentines qui questionne la légitimité de l'ancrage porteño du tango. Certains acteurs politiques ont donc senti la nécessité de repolariser le tango à Buenos Aires, en mobilisant différentes stratégies, telles que l'inscription du tango à l'Unesco, le développement d'un marketing territorial, et une politique méga-événementielle. En effet, les politiques publiques, mais aussi de multiples acteurs privés gravitent autour de cette économie touristique du tango à Buenos Aires. Leur enjeu consiste à accroître la visibilité du tango dans la métropole et vers le monde dans un mouvement à la fois centripète et centrifuge.

Toutefois, une tension apparaît entre les politiques locales visant à renforcer l'attractivité de la ville et certaines initiatives privées, plus polycentriques et diverses, micro-entrepreneuriales et globalisées. Certains acteurs s'inscrivent en décalage (parce que visant d'autres publics ou mobilisant une autre idée du tango) voire en résistance (en revendiquant un tango social) aux logiques perçues comme surtout marchandes et stratégiques des grandes compétitions (Mundial du tango) ou des tango shows. Les géographies de ces différentes formes de tango s'enchevêtrent: le festival de Boedo revendiquant une dimension locale et non marchande se déroule à quelques pas d'une casa de tango emblématique ; milongas, cours de tango, tango shows, sont localisés dans les mêmes quartiers : ce sont des micro-géographies qui se dessinent. Ces décalages et résistances ne s'inscrivent pas de façon linéaire dans une opposition entre logiques de l'ancrage et logiques de la circulation, ou entre logiques localisées et logiques transnationales. Ils ne conduisent pas non plus à opposer tango touristique et tango local: les touristes et danseurs du monde entier constituent à la fois le public et un élément de la scène des tangos à Buenos Aires.

Enfin, cette capitalisation du tango à Buenos Aires entre en concurrence avec un univers du tango davantage polycentrique, à la fois internationalement et nationalement, avec le développement d'un marché du tango dans l'intérieur du pays. L'un des cas exemplaires est la création de l'ETI, encuentro tanguero del interior, qui permet, grâce à un package touristique, à plus de 2000 participants de se retrouver deux fois par an dans plus de 21 villes différentes depuis 2009. Buenos Aires serait alors non plus le cœur mais une étape de la boucle, un noeud symbolique et important, mais non plus la Mecque du tango. 


\section{BIBLIOGRAPHIE}

Apprill, Christophe, « Les métamorphoses d'un havane noir et juteux... », Volume!, 2011, p. 41-67.

Buenos Aires Ciudad, Plan Estratégico de Turismo 2011-2015, 70 p., 2011.

Apprill, Christophe, Les audaces du tango, Petites variations sur la danse et la sensualité, Transboréal, petite philosophie du voyage, 89 p., 2012.

Broclain, Elsa, « Tango ${ }^{\oplus}$. Enjeux d'une stratégie de promotion territoriale fondée sur la

réappropriation d'un patrimoine musical », Questions de communication, 2012, p. 123-140.

Cooper, Artemis, « Tangomanie en Europe et en Amérique du Nord, 19131914 », in Collier Simon, Cooper Artemis, Azzi Maria Suzana, Richard Martin, Tango !, Ed. de la Martinière, 1995.

Debarbieux, Bernard, Del Biaggio, Cristina et Petite, Mathieu, « Spatialités et territorialités du tourisme », Civilisations, n. 57, 2008, p. 75-89.

Debarbieux, Bernard, « Enracinement - Ancrage - Amarrage : raviver les métaphores », L'Espace géographique, vol. tome 43, no. 1, 2014, p. 68-80.

Denigot, Gwen-Haël, Mingalon, Jean-Louis, Honorin, Emmanuelle, Dictionnaire passionné du tango, Seuil, 2015.

Denigot, Gwen-Haël, « Con leyendas que se cantan como tangos... Clichés et stéréotypes de l'histoire du tango ", colloque international Le tango, de Carlos Gardel à Astor Piazzolla : cent années mythiques de créations, Université d'Arras, 2017.

D'Iugo, Marvin, « Aural identity, genealogies of sound technologies and hispanic transnationality on screen », in Durovicova Natasa, Newman Kathleen, World Cinemas. Transnational perspectives, Routledge, 2009.

Duhamel, Philippe, Géographie du tourisme et des loisirs, Dynamiques, acteurs, territoires, Armand Colin, Paris, 2018.

Ente de turismo, Gobierno de la Ciudad Autonoma de Buenos Aires, Anuario Turismo 2016, 2017.

Ferrer, Horacio, El libro del tango (3 tomes), 1980.

Fitch, Melissa, Global Tangos, Travels in Transnational Imaginary, Bucknell University Press, 2015.

Gómez Schettini, Mariana, Almirón, Analía, González Bracco, Mercedes, « La cultura como recurso turistico de las ciudades, el caso de la patrimonialización del tango en Buenos Aires », Estudios y Perspectivas en Turismo, vol. 20, núm. 5, 2011, p. 1027-1046.

Gorelik, Adrian, « Modelos para armar : Buenos Aires, de la crisis al boom », Punto de Vista no ${ }^{\circ 4}$, Buenos Aires, 2006.

Gravari-Barbas, Maria, « Tourism as a heritage producing machine », Tourism Management Perspectives, vol. 26, 2018, p. 5-8.

Lacarrieu Mónica, « Touristes et «non-touristes » dans le monde de l'interculturalité. Un regard à partir du patrimoine immatériel », Autrepart 2006/4 (n40), p. 131-150.

Matallana, Andrea, « Viajeros porteños : músicos y bailarines en New York, 1905-1935 », communication du colloque Pensar, escuchar y representar el Tango, Alliance Française de Buenos Aires, 25/04/2013.

Ministerio de Turismo, Anuario Estadistico de Turismo 2015, 244 p, 2016. 
Morel Hernán, « El giro patrimonial del tango : políticas oficiales, turismo y campeonatos de baile en la ciudad de Buenos Aires ", Cuadernos de Antropología Social, 30, 2009, p. 145-162.

OIC (Observatorio de Industrias Culturales de la Ciudad de Buenos Aires) - Marchini J., El Tango en la Economia de la Ciudad de Buenos Aires, 170 p, 2007.

Patrix, Pénélope, Imaginaire des bas-fonds et poétique « canaille » dans la chanson urbaine. Le fado et le tango, des marges au patrimoine immatériel, Thèse de doctorat, Université de Paris 7, 2014.

Pelinski, Ramon, « Tango nómade. Una metáfora de la globalización. » dans Lencina, Teresita, Garcia Brunelli, Omar, Salton, Ricardo (dir.) Escritos sobre tango. En el Río de la Plata y en la diáspora. Buenos Aires, Centro'feca Ediciones, 2009, p. 65-128.

Pujol, Sergio, Historia del baile : de la milonga a la disco, Buenos Aires, Emecé, 1999.

Salas, Horacio, Le tango, ed. Actes Sud Babel, 1989.

Törnqvist Maria, Tourism and the Globalization of Emotions, The intimate Economy of Tango, Routledge Advances in Feminist Studies and Intersectionnality, 2013.

WTO, Argentina, Basic indicators, 2012-2016, 2017.

Zalko, Nardo, Un siècle de tango, Paris-Buenos Aires, éditions du félin, Poche, 2016.

Presse :

“Tango, bailando en el nuevo siglo”, La Nacion, Buenos Aires, 17/03/2002, https://

www.lanacion.com.ar/212263-tango-bailando-en-el-nuevo-siglo

"El tango, más cerca de ser patrimonio de la humanidad", El Mundo, Buenos Aires, 12/08/2009, http://www.elmundo.es/elmundo/2009/08/12/cultura/1250070778.html

Webographie :

Tangotecnia, Los mercados de turismo de tango, el potencial de Brasil, México, Chile, 16 p. 2017. https://tangotecnia.wordpress.com/los-mercados-de-turismo-de-tango-2017/

Hoymilonga, https://www.hoy-milonga.com/buenos-aires/fr

\section{NOTES}

1. Le terme milonga désigne à la fois le bal tango et le lieu et moment de son déroulé (Denigot, Mingalon, Honorin, 2015)

2. Recherche financée par l'ANR, pour le projet Patrimondi, dirigé par Maria Gravari-Barbas, cf : https://patrimondi.hypotheses.org/. Les entretiens sont été réalisés en 2016, 2017 et 2018.

3. Cette catégorie de visiteurs englobe les touristes (qui passent au moins une nuitée sur le territoire argentin) et les excursionnistes.

4. Ces enquêtes sont conduites durant le Mondial depuis l'édition 2004, mais elles ne sont pas conçues de la même façon, rendant parfois difficile la comparaison entre éditions.

5. Les différentes enquêtes sont disponibles à cette adresse : https://tangotecnia.wordpress.com/

6. Les guides Gallimard (Cartoville), Le Petit Futé, Le Routard, Lonely Planet, Michelin, National Geographic.

7. L'association à Montevideo est généralement justifiée par l'apport de musiciens uruguayens à l'histoire du tango, avec pour événement majeur la composition à Montevideo d'un des tangos emblématiques, La Cumparsita. Toutefois, ces dernières années, la municipalité de Montevideo souhaite réinvestir le tango, à l'exemple de Buenos Aires. Cela n'a pas constitué un axe de nos enquêtes. 
8. C'est pour ce motif qu'une première candidature à l'Unesco sera d'ailleurs rejetée : en 2001, dans le cadre de l'inscription aux Chefs d'œuvres du Patrimoine oral et immatériel de l'humanité, l'Unesco privilégiait les pratiques culturelles nécessitant des mesures de sauvegarde, ce qui n'est pas le cas du tango.

9. Une milonga centenaire du quartier de Villa Urquiza connue comme la "Cathédrale du tango" traditionnel.

\section{RÉSUMÉS}

Le tango, né dans le bassin du Rio de la Plata entre Buenos Aires et Montevideo à la fin du $\mathrm{XIX}^{\mathrm{e}}$ siècle est une musique, une danse, une culture qui a été diffusée très tôt dans les salons de la Belle Époque. Aujourd'hui, le tango se danse dans le monde entier et devient une des motivations du tourisme à Buenos Aires. L'inscription du tango comme PCI (patrimoine culturel immatériel) en 2009 marque une volonté de légitimer les origines du tango et de soutenir une activité touristique en plein essor depuis les années 2000. Les acteurs économiques, touristiques et patrimoniaux cherchent à ré-ancrer le tango à Buenos Aires en même temps que s'opère un mouvement d'extension vers le monde des tango shows et de la pratique de la danse. Buenos Aires est-elle encore, comme l'affirment les acteurs publics la capitale du tango?

El tango, nacido en la cuenca del Río de la Plata entre Buenos Aires y Montevideo a fines del siglo XIX es una música, una danza que fue difundida muy temprano en los salones de la Belle Epoque. Hoy el tango se baila en todo el mundo y se convierte en uno de los motivos del turismo en Buenos Aires. La inscripción del tango como PCI (patrimonio cultural inmaterial) en 2009 marca la voluntad de legitimar los orígenes del tango y apoyar una actividad turística en pleno apogeo desde la década del 2000. Los actores económicos, turísticos y patrimoniales buscan volver a anclar el tango en Buenos Aires al mismo tiempo como un movimiento de extensión al mundo de los espectáculos de tango y la práctica de la danza. ¿Sigue siendo Buenos Aires, como dicen los actores públicos, la capital del tango?

The tango originated in the Rio de la Plata Basin between Buenos Aires and Montevideo in the late nineteenth century and is made of a plurality of cultural practices among which musical performances and dancing that have spread very early on across Europe during the Belle Epoque. Today tango is practiced around the world and has become one of the main tourist attractions in Buenos Aires. The inscription of tango as Intangible Cultural Heritage in 2009 marks a will to legitimize the origins of tango and support a growing tourism industry since the 2000s. The different actors from the economic and the tourism fields as well as the cultural heritage one seek to re-anchor tango in Buenos Aires while tango keeps expanding and is globally practiced by musicians and dancers. Is Buenos Aires, as the public actors claim, the world's capital of tango?

\section{INDEX}

Keywords : Tango, Buenos Aires, UNESCO, turismo, patrimonio, políticas públicas

Mots-clés : Tango, Buenos Aires, UNESCO, tourisme, patrimoine, politiques publiques 


\section{AUTEURS}

\section{LINDA BOUKHRIS}

Maîtresse de Conférences en géographie, Université Paris I Panthéon-Sorbonne/IREST, membre du laboratoire EA 7337-EIREST (Equipe interdisciplinaire de Recherche Sur le Tourisme)

\section{ELSA BROCLAIN}

Doctorante au Centre de Recherches sur les Arts et le Langage (CRAL EHESS-CNRS)

\section{SÉBASTIEN JACQUOT}

Maître de Conférences en géographie, Université Paris I Panthéon-Sorbonne/IREST, membre du laboratoire EA 7337-EIREST (Equipe interdisciplinaire de Recherche Sur le Tourisme) et associé à l'UMR PRODIG

\section{ELODIE SALIN}

Maîtresse de Conférences en géographie, Le Mans Université, Laboratoire Espaces et Sociétés (UMR ESO 6590 CNRS), Chercheuse associée EIREST (Equipe interdisciplinaire de Recherche Sur le Tourisme) - Université Paris I Panthéon-Sorbonne 